\title{
The Marshall-Olkin Right Truncated Fréchet-Inverted Weibull Distribution: Its Properties and Applications
}

\author{
Nora Nader$^{1}$, M. A. El-Damcese ${ }^{2}$, B. S. El-Desouky ${ }^{1}$ \\ ${ }^{1}$ Department of Mathematics, Faculty of Science, Mansoura University, Mansoura, Egypt \\ ${ }^{2}$ Department of Mathematies, Faculty of Science, Tanta University, Tanta, Egypt \\ Email: sci_nora2012@yahoo.com,meldamcese@yahoo.com,b_desouky@yahoo.com
}

How to cite this paper: Nader, N., El-Damcese, M.A. and El-Desouky, B.S. (2021) The Marshall-Olkin Right Truncated Fréchet-Inverted Weibull Distribution: Its Properties and Applications. Open Journal of Modelling and Simulation, 9, 74-89.

https://doi.org/10.4236/ojmsi.2021.91005

Received: September 30, 2020

Accepted: January 24, 2021

Published: January 27, 2021

Copyright $\odot 2021$ by author(s) and Scientific Research Publishing Inc. This work is licensed under the Creative Commons Attribution International License (CC BY 4.0).

http://creativecommons.org/licenses/by/4.0/

\begin{abstract}
In this paper, a new probability distribution is proposed by using Marshall and Olkin transformation. Some of its properties such as moments, moment generating function, order statistics and reliability functions are derived. The method of maximum likelihood is used to estimate the model parameters. The graphs of the reliability function and hazard rate function are plotted by taken some values of the parameters. Three real life applications are introduced to compare the behaviour of the new distribution with other distributions.
\end{abstract}

\section{Keywords}

Marshall and Olkin, Moment Generating Function, Density Function, Order Statistics, Reliability Function, Inverse Weibull Distribution, Maximum Likelihood

\section{Introduction}

In the past, researchers highlighted on the inversion of univariate probability models. They have applied the inverse technique for many distributions. For example, there are many examples such as inverted beta [1], inverse Rayleigh [2], inverse Gaussian [3], inverse Weibull [4], inverted Burr type XII also called Burr type III [5], inverted exponential [6] and many other distributions. The Weibull distribution is a continuous probability distribution which identified by Fréchet [7]. Inverse Weibull distribution has two parameters $\alpha$ and $\beta$ with probability density function of a random variable $X$ is denoted by

$$
G(x)=\mathrm{e}^{\left(-\alpha x^{-\beta}\right)}, x \geq 0, \alpha>0, \beta>0
$$


The basic objective in this paper is the study how the Marshall and Olkin right truncated Fréchet-Inverted Weibull distribution applied. Later, Haq [8] used this conversion to improve Marshall-Olkin length biased moment exponential distribution. Marshall and Olkin [9] proposed an ingenious approach for adding an additional shape parameter to the existing distribution. So, we find the cumulative distribution function $F(x)$ of right truncated Fréchet-inverted Weibull distribution (RTFIWD)

$$
F(x)=\frac{G(x)}{G(b)}=\mathrm{e}^{-\alpha\left(x^{-\beta}-b^{-\beta}\right)}, 0<x \leq b, \alpha>0, \beta>0 .
$$

The pdf $f(x)$ of (RTFIWD) is

$$
f(x)=\frac{\mathrm{d} F(x)}{\mathrm{d} x}=\frac{\alpha \beta}{x^{\beta+1}} \mathrm{e}^{-\alpha\left(x^{-\beta}-b^{-\beta}\right)}, 0<x \leq b, \alpha>0, \beta>0,
$$

the reliability function $R(x)$ (the survival function) of the Marshall and Olkin (MO) family is defined by

$$
\begin{gathered}
R(x)=\frac{\gamma \bar{F}(X)}{1-\bar{\gamma} \bar{F}(X)}, \gamma>0 \\
\bar{\gamma}=1-\gamma,
\end{gathered}
$$

using (2) into (4), we obtain $R(x)$ by the Marshall and Olkin right truncated Fréchet-inverted Weibull distribution (MORTFIWD) as

$$
R(x)=\frac{\gamma\left(1-\mathrm{e}^{-\alpha\left(x^{-\beta}-b^{-\beta}\right)}\right)}{1-\bar{\gamma}\left(1-\mathrm{e}^{-\alpha\left(x^{-\beta}-b^{-\beta}\right)}\right)}, \gamma>0 .
$$

The pdf corresponding to (5) is given by

$$
\begin{aligned}
g(x) & =\frac{-\mathrm{d} R(x)}{\mathrm{d} x}=\frac{\gamma \bar{F}(X)}{1-\bar{\gamma} \bar{F}(X)}, \gamma>0 \\
& =\frac{\gamma \alpha \beta \mathrm{e}^{-\alpha\left(x^{-\beta}-b^{-\beta}\right)}}{x^{\beta+1}\left[1-\bar{\gamma}\left(1-\mathrm{e}^{-\alpha\left(x^{-\beta}-b^{-\beta}\right)}\right)\right]^{2}}, 0<x \leq b, \alpha>0, \beta>0 ; \gamma>0,
\end{aligned}
$$

and its hazard function reduces to

$$
h(x)=\frac{g(x)}{R(x)}=\frac{\alpha \beta \mathrm{e}^{-\alpha\left(x^{-\beta}-b^{-\beta}\right)}}{x^{\beta+1}\left[1-\mathrm{e}^{-\alpha\left(x^{-\beta}-b^{-\beta}\right)}\right]\left[1-\bar{\gamma}\left(1-\mathrm{e}^{-\alpha\left(x^{-\beta}-b^{-\beta}\right)}\right)\right]} .
$$

The cumulative distribution function $F(x)$ of the Marshall and Olkin of the right truncated Fréchet-inverted Weibull distribution (MORTFIWD) is denoted by

$$
F(x)=1-R(x),
$$

by substituting (5) in (8), we find 


$$
\begin{aligned}
& F(x)=1-\frac{\gamma\left(1-\mathrm{e}^{-\alpha\left(x^{-\beta}-b^{-\beta}\right)}\right)}{1-\bar{\gamma}\left(1-\mathrm{e}^{-\alpha\left(x^{-\beta}-b^{-\beta}\right)}\right)} \\
&=\frac{\left(1-\bar{\gamma}\left(1-\mathrm{e}^{-\alpha\left(x^{-\beta}-b^{-\beta}\right)}\right)\right)-\gamma\left(1-\mathrm{e}^{-\alpha\left(x^{-\beta}-b^{-\beta}\right)}\right)}{1-\bar{\gamma}\left(1-\mathrm{e}^{-\alpha\left(x^{-\beta}-b^{-\beta}\right)}\right)} \\
&=\frac{1-\bar{\gamma}\left(1-\mathrm{e}^{-\alpha\left(x^{-\beta}-b^{-\beta}\right)}\right)-\gamma\left(1-\mathrm{e}^{-\alpha\left(x^{-\beta}-b^{-\beta}\right)}\right)}{1-\bar{\gamma}\left(1-\mathrm{e}^{-\alpha\left(x^{-\beta}-b^{-\beta}\right)}\right)} \\
&=\frac{1-\left(1-\mathrm{e}^{-\alpha\left(x^{-\beta}-b^{-\beta}\right)}\right)(\bar{\gamma}+\gamma)}{1-\bar{\gamma}\left(1-\mathrm{e}^{-\alpha\left(x^{-\beta}-b^{-\beta}\right)}\right)} \\
&=\frac{1-\left(1-\mathrm{e}^{-\alpha\left(x^{-\beta}-b^{-\beta}\right)}\right)(1-\gamma+\gamma)}{1-\bar{\gamma}\left(1-\mathrm{e}^{-\alpha\left(x^{-\beta}-b^{-\beta}\right)}\right)} \\
&=\frac{1-\left(1-\mathrm{e}^{-\alpha\left(x^{-\beta}-b^{-\beta}\right)}\right)}{1-\bar{\gamma}\left(1-\mathrm{e}^{-\alpha\left(x^{-\beta}-b^{-\beta}\right)}\right)} \\
& \mathrm{e}^{-\alpha\left(x^{-\beta}-b^{-\beta}\right)} \\
& 1-\bar{\gamma}\left(1-\mathrm{e}^{-\alpha\left(x^{-\beta}-b^{-\beta}\right)}\right)
\end{aligned}
$$

Figure 1 and Figure 2 outlined the manner of the density function and interprets the susctability and elasticity of the pattern graphically. The pdf plot shows that for some different values $\beta$. Figure 3 and Figure 4 outlined the manner of

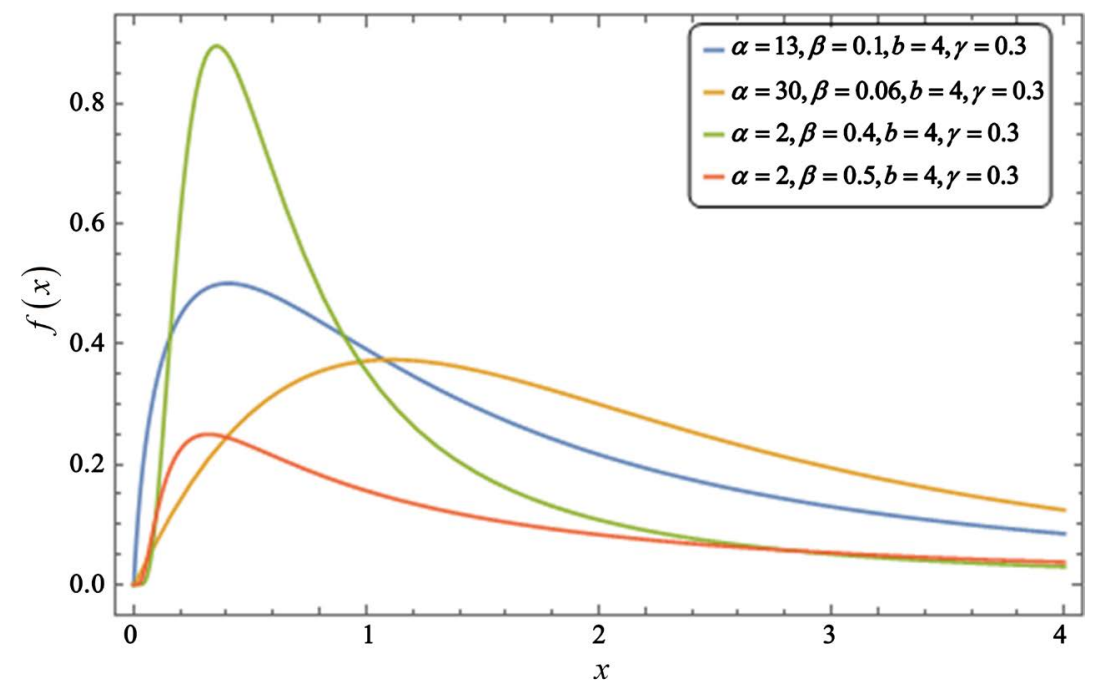

Figure 1. The density function for different values of the parameters. 


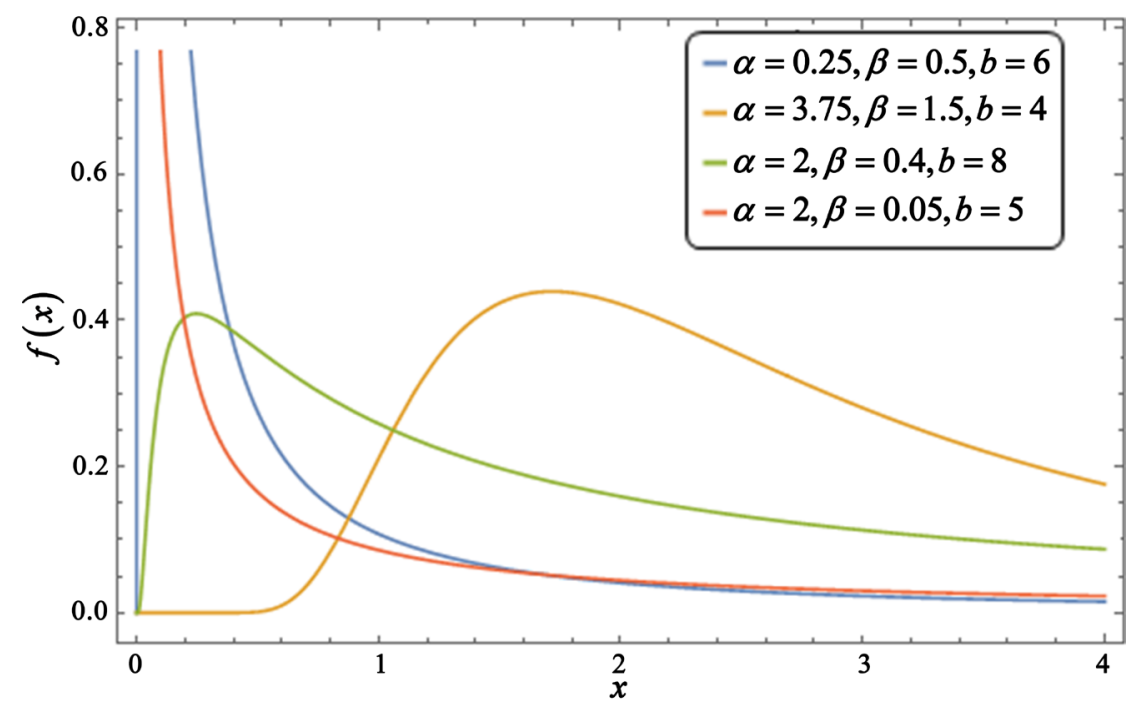

Figure 2. The density function for different values of the parameters.

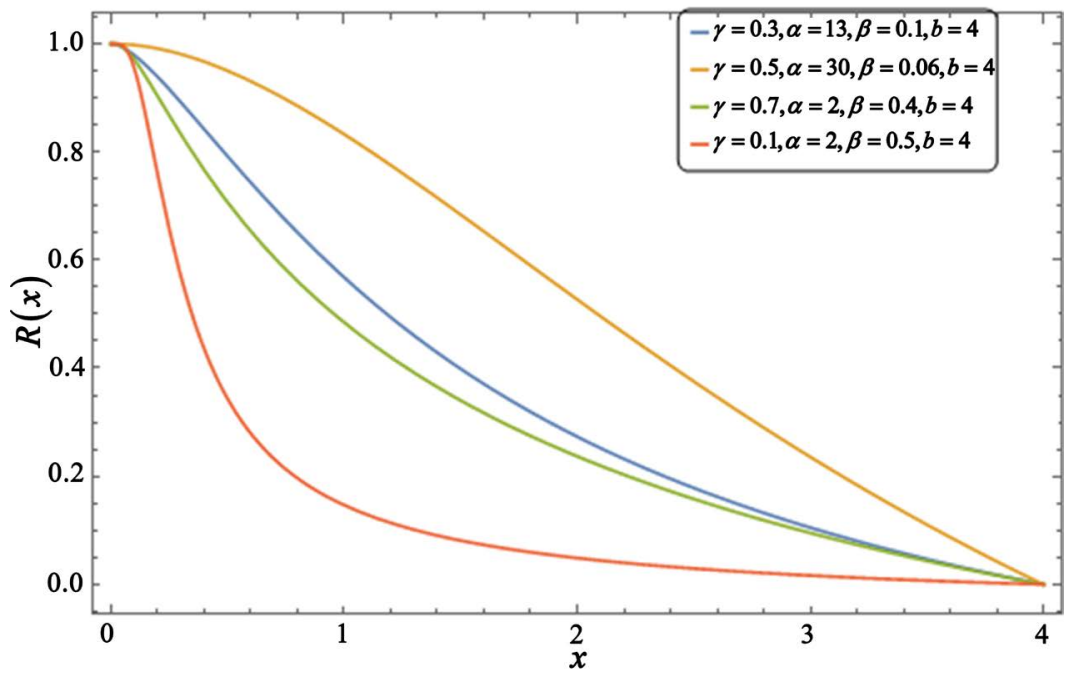

Figure 3. The hazard rate function for different values of the parameters.

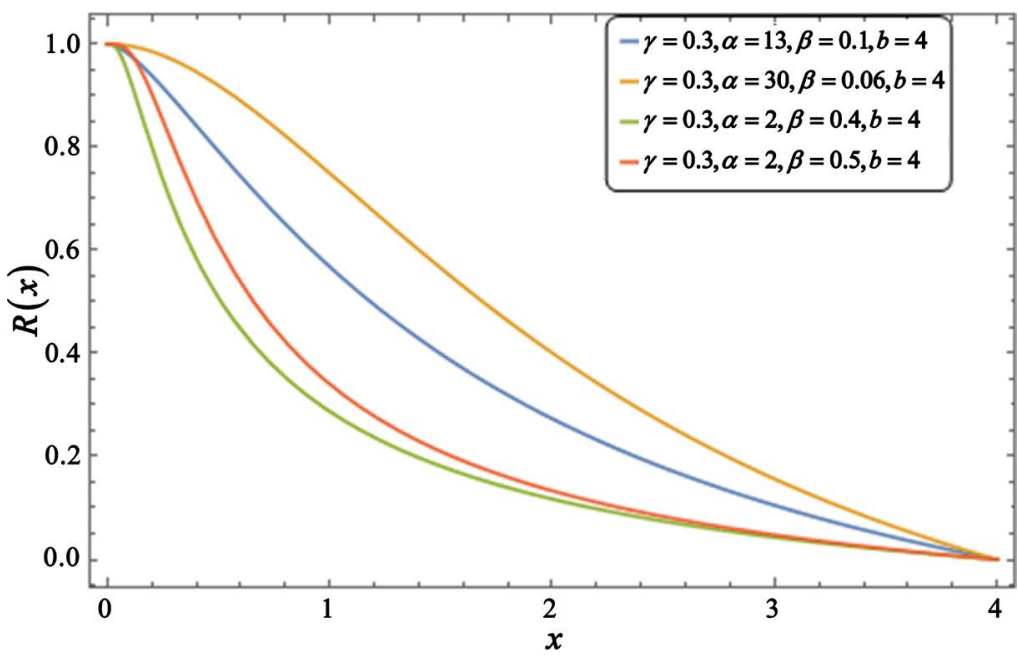

Figure 4. The hazard rate function for different values of the parameters. 
hazard rate function. Figure 5 and Figure 6 outlined the manner of reserved hazard rate function which is monocular at different parameter combinations.

\section{Moments}

The $r$ th moments $\mu_{r}^{\prime}$ of MORTFIWD

$$
\begin{aligned}
& \mu_{r}^{\prime}=E\left(x^{r}\right)=\int_{0}^{b} x^{r} \cdot g(x) \mathrm{d} x \\
& =\int_{0}^{b} x^{r} \cdot \frac{\gamma \alpha \beta \mathrm{e}^{-\alpha\left(x^{-\beta}-b^{-\beta}\right)}}{x^{\beta+1}\left[1-\bar{\gamma}\left(1-\mathrm{e}^{-\alpha\left(x^{-\beta}-b^{-\beta}\right)}\right)\right]^{2}} \mathrm{~d} x \\
& =\int_{0}^{b} x^{r-\beta-1} \cdot \gamma \alpha \beta \mathrm{e}^{-\alpha\left(x^{-\beta}-b^{-\beta}\right)\left[1-\bar{\gamma}\left(1-\mathrm{e}^{-\alpha\left(x^{-\beta}-b^{-\beta}\right)}\right)\right]^{-2}} \mathrm{~d} x,
\end{aligned}
$$

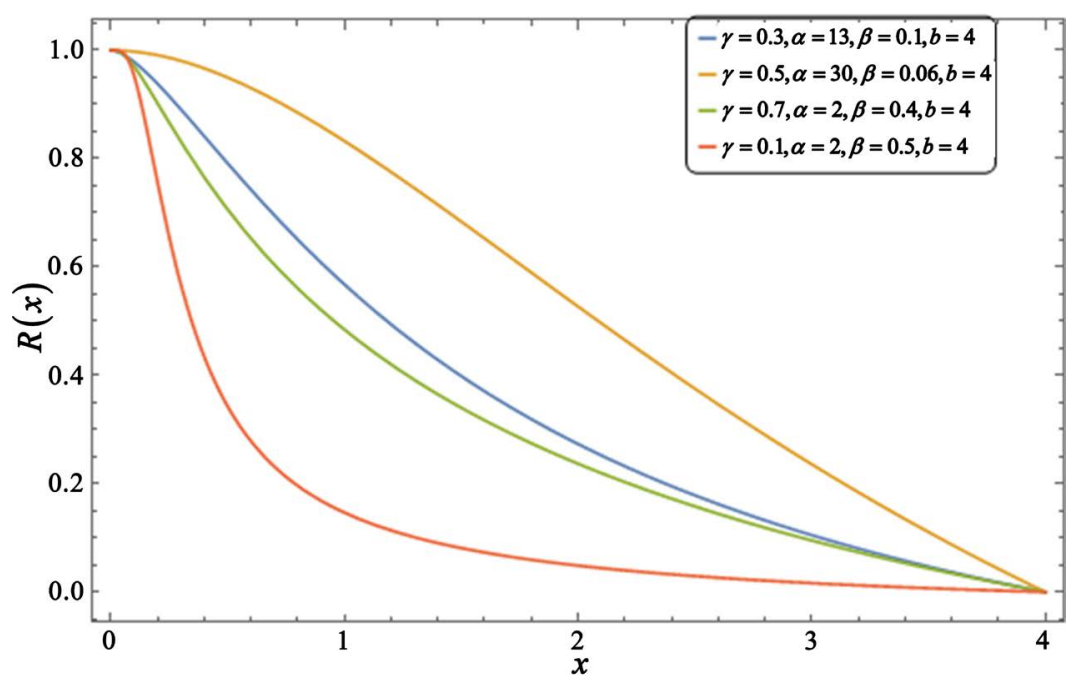

Figure 5. The reserved hazard rate function for different values of the parameters.

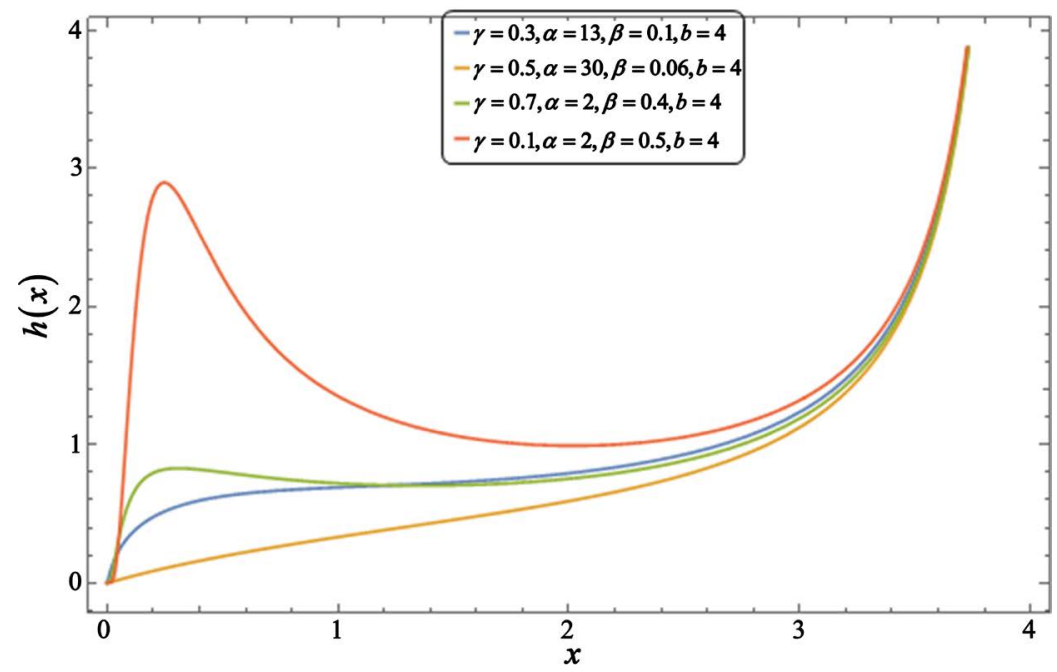

Figure 6. The reserved hazard rate function for different values of the parameters. 
since $0<\left[1-\bar{\gamma}\left(1-\mathrm{e}^{-\alpha\left(x^{-\beta}-b^{-\beta}\right)}\right)\right]^{2}<1$, for $x>0$ we have

$$
\left[1-\bar{\gamma}\left(1-\mathrm{e}^{-\alpha\left(x^{-\beta}-b^{-\beta}\right)}\right)\right]^{-2}=\sum_{i=0}^{\infty}(i+1) \bar{\gamma}^{i}\left(1-\mathrm{e}^{-\alpha\left(x^{-\beta}-b^{-\beta}\right)}\right)^{i},
$$

by substituting (11) in (10), we find

$$
\begin{aligned}
\mu_{r}^{\prime} & =\int_{0}^{b} x^{r-\beta-1} \cdot \gamma \alpha \beta \mathrm{e}^{-\alpha\left(x^{-\beta}-b^{-\beta}\right)\left[1-\bar{\gamma}\left(1-\mathrm{e}^{-\alpha\left(x^{-\beta}-b^{-\beta}\right)}\right)\right]} \mathrm{d} x \\
& =\int_{0}^{b} x^{r-\beta-1} \cdot \gamma \alpha \beta \mathrm{e}^{-\alpha\left(x^{-\beta}-b^{-\beta}\right)} \sum_{j=0}^{\infty}(j+1) \bar{\gamma}^{j}\left(1-\mathrm{e}^{-\alpha\left(x^{-\beta}-b^{-\beta}\right)}\right)^{j} \mathrm{~d} x \\
& =\sum_{j=0}^{\infty}(j+1) \bar{\gamma}^{j} \cdot \gamma \alpha \beta \int_{0}^{b} x^{r-\beta-1} \mathrm{e}^{-\alpha\left(x^{-\beta}-b^{-\beta}\right)}\left(1-\mathrm{e}^{-\alpha\left(x^{-\beta}-b^{-\beta}\right)}\right)^{j} \mathrm{~d} x \\
& =\gamma \alpha \beta \sum_{i=0}^{\infty}(j+1) \bar{\gamma}^{j} \cdot \phi_{r, b}(x),
\end{aligned}
$$

where

$$
\begin{aligned}
\phi_{r, b}(x) & =\int_{0}^{b} x^{r-\beta-1} \mathrm{e}^{-\alpha\left(x^{-\beta}-b^{-\beta}\right)}\left(1-\mathrm{e}^{-\alpha\left(x^{-\beta}-b^{-\beta}\right)}\right)^{j} \mathrm{~d} x \\
\phi_{r, b}(x) & =\int_{0}^{b} x^{r-\beta-1} \mathrm{e}^{-\alpha\left(x^{-\beta}-b^{-\beta}\right)} \sum_{i=0}^{\infty}\left(\begin{array}{l}
j \\
i
\end{array}\right)(-1)^{i} \mathrm{e}^{-\alpha i\left(x^{-\beta}-b^{-\beta}\right)} \mathrm{d} x \\
= & \sum_{i=0}^{\infty}\left(\begin{array}{l}
j \\
i
\end{array}\right)(-1)^{i} \int_{0}^{b} x^{r-\beta-1} \mathrm{e}^{-\alpha(i+1)\left(x^{-\beta}-b^{-\beta}\right)} \mathrm{d} x \\
= & \sum_{i=0}^{\infty}\left(\begin{array}{l}
j \\
i
\end{array}\right)(-1)^{i} \int_{0}^{b} x^{r-\beta-1} \mathrm{e}^{-\alpha(i+1) x^{-\beta}} \mathrm{e}^{\alpha(i+1) b^{-\beta}} \mathrm{d} x \\
= & \sum_{i=0}^{\infty}\left(\begin{array}{l}
j \\
i
\end{array}\right)(-1)^{i} \mathrm{e}^{\alpha(i+1) b^{-\beta}} \int_{0}^{b} x^{r-\beta-1} \mathrm{e}^{-\alpha(i+1) x^{-\beta}} \mathrm{d} x .
\end{aligned}
$$

Setting

$$
\begin{gathered}
-\alpha(i+1) x^{-\beta}=t \\
x^{-\beta}=\frac{1}{\alpha(i+1)} t \\
x=\left(\frac{1}{\alpha(i+1)}\right)^{\frac{-1}{\beta}} t^{\frac{-1}{\beta}} \\
\mathrm{d} x=\left(\frac{1}{\alpha(i+1)}\right)^{\frac{-1}{\beta}}\left(\frac{-1}{\beta}\right) t^{\frac{-1}{\beta}-1} \mathrm{~d} t \\
x=0 \rightarrow t=0 \\
x=b \rightarrow t=\alpha(i+1) b^{-\beta},
\end{gathered}
$$

we find (14) becomes as follows 


$$
\begin{aligned}
& \phi_{r, b}(x)=\sum_{i=0}^{\infty}\left(\begin{array}{l}
j \\
i
\end{array}\right)(-1)^{i} \mathrm{e}^{\alpha(i+1) b^{-\beta}} \int_{0}^{b} x^{r-\beta-1} \mathrm{e}^{-\alpha(i+1) x^{-\beta}} \mathrm{d} x \\
& =\sum_{i=0}^{\infty}\left(\begin{array}{l}
j \\
i
\end{array}\right)(-1)^{i} \mathrm{e}^{\alpha(i+1) b^{-\beta}} \int_{0}^{\alpha(i+1) b^{-\beta}}\left(\left(\frac{1}{\alpha(i+1)}\right)^{\frac{-1}{\beta}} t^{\frac{-1}{\beta}}\right)^{r-\beta-1} \\
& \times \mathrm{e}^{-t}\left(\frac{1}{\alpha(i+1)}\right)^{\frac{-1}{\beta}}\left(\frac{-1}{\beta}\right) t^{\frac{-1}{\beta}-1} \mathrm{~d} t \\
& =\sum_{i=0}^{\infty}\left(\begin{array}{l}
j \\
i
\end{array}\right)(-1)^{i} \mathrm{e}^{\alpha(i+1) b^{-\beta}}\left(\left(\frac{1}{\alpha(i+1)}\right)^{\frac{-1}{\beta}}\right)^{r-\beta-1}\left(\frac{1}{\alpha(i+1)}\right)^{\frac{-1}{\beta}} \\
& \times\left(\frac{-1}{\beta}\right)^{\alpha(i+1) b^{-\beta}}\left(t^{\frac{-1}{\beta}}\right)^{r-\beta-1} t^{\frac{-1}{\beta}-1} \mathrm{e}^{-t} \mathrm{~d} t \\
& =\sum_{i=0}^{\infty}\left(\begin{array}{l}
j \\
i
\end{array}\right)(-1)^{i} \mathrm{e}^{\alpha(i+1) b^{-\beta}}\left(\left(\frac{1}{\alpha(i+1)}\right)^{\frac{-1}{\beta}}\right)^{r-\beta-1}\left(\frac{1}{\alpha(i+1)}\right)^{\frac{-1}{\beta}} \\
& \times\left(\frac{-1}{\beta}\right)^{\alpha(i+1) b^{-\beta}} t^{\frac{-r+\beta}{\beta}-1} \mathrm{e}^{-t} \mathrm{~d} t \\
& =\sum_{i=0}^{\infty}\left(\begin{array}{l}
j \\
i
\end{array}\right)(-1)^{i} \mathrm{e}^{\alpha(i+1) b^{-\beta}}\left(\left(\frac{1}{\alpha(i+1)}\right)^{\frac{-1}{\beta}}\right)^{r-\beta-1}\left(\frac{1}{\alpha(i+1)}\right)^{\frac{-1}{\beta}} \\
& \times\left(\frac{-1}{\beta}\right)\left(\alpha(i+1) b^{-\beta}\right)^{\frac{-r+\beta}{\beta}} \Gamma\left(\frac{-r+\beta}{\beta}\right) \mathrm{e}^{\alpha(i+1) b^{-\beta}} \\
& \times \sum_{k=0}^{\infty} \frac{\left(\alpha(i+1) b^{-\beta}\right)^{k}}{\Gamma\left(\frac{-r+\beta}{\beta}+\alpha(i+1) b^{-\beta}+1\right)},
\end{aligned}
$$

where

$$
\begin{aligned}
& \int_{0}^{\alpha(i+1) b^{-\beta}} t^{\frac{-r+\beta}{\beta}-1} \mathrm{e}^{-t} \mathrm{~d} t \\
= & \left(\alpha(i+1) b^{-\beta}\right)^{\frac{-r+\beta}{\beta}} \Gamma\left(\frac{-r+\beta}{\beta}\right) \mathrm{e}^{\alpha(i+1) b^{-\beta}} \sum_{k=0}^{\infty} \frac{\left(\alpha(i+1) b^{-\beta}\right)^{k}}{\Gamma\left(\frac{-r+\beta}{\beta}+\alpha(i+1) b^{-\beta}+1\right)},
\end{aligned}
$$

by using the definition of the incomplete gamma function

$$
\gamma(s, x)=\int_{0}^{x} t^{s-1} \mathrm{e}^{-t} \mathrm{~d} t=x^{s} \Gamma(s) \mathrm{e}^{-x} \sum_{k=0}^{\infty} \frac{x^{k}}{\Gamma(s+x+1)} .
$$

So we find the $r$ th moments $\mu_{r}^{\prime}$ of MORTFIWD

$$
\mu_{r}^{\prime}=\gamma \alpha \beta \sum_{i=0}^{\infty}(j+1) \bar{\gamma}^{j} \sum_{i=0}^{\infty}\left(\begin{array}{l}
j \\
i
\end{array}\right)(-1)^{i} \mathrm{e}^{\alpha(i+1) b^{-\beta}}\left(\left(\frac{1}{\alpha(i+1)}\right)^{\frac{-1}{\beta}}\right)^{r-\beta-1}
$$




$$
\begin{aligned}
\times\left(\frac{1}{\alpha(i+1)}\right)^{\frac{-1}{\beta}}\left(\frac{-1}{\beta}\right)\left(\alpha(i+1) b^{-\beta}\right)^{\frac{-r+\beta}{\beta}} \Gamma\left(\frac{-r+\beta}{\beta}\right) \mathrm{e}^{\alpha(i+1) b^{-\beta}} \\
\times \sum_{k=0}^{\infty} \frac{\left(\alpha(i+1) b^{-\beta}\right)^{k}}{\Gamma\left(\frac{-r+\beta}{\beta}+\alpha(i+1) b^{-\beta}+1\right)} \\
\mu_{r}^{\prime}=\gamma \alpha \beta \sum_{i=0}^{\infty} \sum_{i=0}^{\infty} \sum_{k=0}^{\infty}(-1)^{i+1}(j+1) \bar{\gamma}^{j}\left(\left(\frac{1}{\alpha(i+1)}\right)^{\frac{-1}{\beta}}\right)^{r-\beta-1} \\
\times\left(\frac{1}{\alpha(i+1)}\right)^{\frac{-1}{\beta}}\left(\alpha(i+1) b^{-\beta}\right)^{\frac{-r+\beta}{\beta}} \\
\quad \times \Gamma\left(\frac{-r+\beta}{\beta}\right) \mathrm{e}^{2 \alpha(i+1) b^{-\beta}}\left(\begin{array}{l}
j \\
i
\end{array}\right) \frac{\left(\alpha(i+1) b^{-\beta}\right)^{k}}{\Gamma\left(\frac{-r+\beta}{\beta}+\alpha(i+1) b^{-\beta}+1\right)}
\end{aligned}
$$

\section{Moment Generting Function}

We need to compute the moment generting function of the Marshall and Olkin of the right truncated Fréchet-inverted Weibull distribution (MORTFIWD). If $X$ a random variable has the distribution MORTFIWD where $\theta=(\alpha, \beta, b)$ and $\gamma$ is a positive integer, then the moment generting function of MORTFIWD is denoted by

$$
\begin{aligned}
M_{x}(t) & =E\left(\mathrm{e}^{t x}\right)=\int_{0}^{b} \mathrm{e}^{t x} \cdot g(x) \mathrm{d} x \\
& =\int_{0}^{b} \mathrm{e}^{t x} \frac{\gamma \alpha \beta \mathrm{e}^{-\alpha\left(x^{-\beta}-b^{-\beta}\right)}}{x^{\beta+1}\left[1-\bar{\gamma}\left(1-\mathrm{e}^{-\alpha\left(x^{-\beta}-b^{-\beta}\right)}\right)\right]^{2}} \mathrm{~d} x \\
& =\int_{0}^{b} x^{-\beta-1} \mathrm{e}^{t x} \gamma \alpha \beta \mathrm{e}^{-\alpha\left(x^{-\beta}-b^{-\beta}\right)\left[1-\bar{\gamma}\left(1-\mathrm{e}^{-\alpha\left(x^{-\beta}-b^{-\beta}\right)}\right)\right]^{-2}} \mathrm{~d} x .
\end{aligned}
$$

From (11), we find

$$
\begin{aligned}
M_{x}(t) & =\sum_{j=0}^{\infty}(j+1) \bar{\gamma}^{j} \cdot \gamma \alpha \beta \int_{0}^{b} x^{-\beta-1} \mathrm{e}^{t x} \mathrm{e}^{-\alpha\left(x^{-\beta}-b^{-\beta}\right)}\left(1-\mathrm{e}^{-\alpha\left(x^{-\beta}-b^{-\beta}\right)}\right)^{j} \mathrm{~d} x \\
& =\sum_{j=0}^{\infty}(j+1) \bar{\gamma}^{j} \cdot \gamma \alpha \beta \Pi_{j}(x),
\end{aligned}
$$

where

$$
\begin{aligned}
& \Pi_{j}(x)=\int_{0}^{b} x^{-\beta-1} \mathrm{e}^{t x} \mathrm{e}^{-\alpha\left(x^{-\beta}-b^{-\beta}\right)}\left(1-\mathrm{e}^{-\alpha\left(x^{-\beta}-b^{-\beta}\right)}\right)^{j} \mathrm{~d} x \\
& \Pi_{j}(x)=\sum_{i=0}^{\infty}\left(\begin{array}{l}
j \\
i
\end{array}\right)(-1)^{i} \mathrm{e}^{\alpha(i+1) b^{-\beta}} \int_{0}^{b} x^{-\beta-1} \mathrm{e}^{t x} \mathrm{e}^{-\alpha(i+1) x^{-\beta}} \mathrm{d} x
\end{aligned}
$$




$$
\begin{aligned}
= & \sum_{i=0}^{\infty}\left(\begin{array}{l}
j \\
i
\end{array}\right)(-1)^{i} \mathrm{e}^{\alpha(i+1) b^{-\beta}} \int_{0}^{\alpha(i+1) b^{-\beta}}\left(\left(\frac{1}{\alpha(i+1)}\right)^{\frac{-1}{\beta}} u^{-\frac{1}{\beta}}\right)^{-\beta-1} \\
& \times \mathrm{e}^{t\left(\frac{1}{\alpha(i+1)}\right)^{\frac{-1}{\beta} u^{\frac{-1}{\beta}}}} \mathrm{e}^{-u}\left(\frac{1}{\alpha(i+1)}\right)^{\frac{-1}{\beta}}\left(\frac{-1}{\beta}\right) u^{\frac{-1}{\beta}-1} \mathrm{~d} u \\
= & \sum_{i=0}^{\infty}\left(\begin{array}{l}
j \\
i
\end{array}\right)(-1)^{i} \mathrm{e}^{\alpha(i+1) b^{-\beta}} \frac{-1}{\alpha \beta(i+1)} \int_{0}^{\alpha(i+1) b^{-\beta}} \mathrm{e}^{-u} \mathrm{e}^{t\left(\frac{1}{\alpha(i+1)}\right)^{\frac{-1}{\beta} u^{\frac{-1}{\beta}}}} \mathrm{d} u \\
= & \sum_{i=0}^{\infty}\left(\begin{array}{l}
j \\
i
\end{array}\right)(-1)^{i} \mathrm{e}^{\alpha(i+1) b^{-\beta}} \frac{-1}{\alpha \beta(i+1)} \int_{0}^{\alpha(i+1) b^{-\beta}} \mathrm{e}^{t\left(\frac{1}{\alpha(i+1)}\right)^{\frac{-1}{\beta} u^{\frac{-1}{\beta}}-u} \mathrm{~d} u .}
\end{aligned}
$$

\section{Inverse Moments}

In this section, inverse moments

$$
\begin{aligned}
& \mu_{r^{-1}}^{\prime}=E\left(\frac{1}{x^{r}}\right)=\int_{0}^{b} \frac{1}{x^{r}} \cdot g(x) \mathrm{d} x \\
& =\int_{0}^{b} \frac{1}{x^{r}} \cdot \frac{\gamma \alpha \beta \mathrm{e}^{-\alpha\left(x^{-\beta}-b^{-\beta}\right)}}{x^{\beta+1}\left[1-\bar{\gamma}\left(1-\mathrm{e}^{-\alpha\left(x^{-\beta}-b^{-\beta}\right)}\right)\right]^{2}} \mathrm{~d} x \\
& =\int_{0}^{b} x^{-r-\beta-1} \cdot \gamma \alpha \beta \mathrm{e}^{-\alpha\left(x^{-\beta}-b^{-\beta}\right)}\left[1-\bar{\gamma}\left(1-\mathrm{e}^{-\alpha\left(x^{-\beta}-b^{-\beta}\right)}\right)\right]^{-2} \mathrm{~d} x,
\end{aligned}
$$

substituting by (11) in (10), we have

$$
\begin{aligned}
\mu_{r^{-1}}^{\prime} & =\int_{0}^{b} x^{-r-\beta-1} \cdot \gamma \alpha \beta \mathrm{e}^{-\alpha\left(x^{-\beta}-b^{-\beta}\right)\left[1-\bar{\gamma}\left(1-\mathrm{e}^{-\alpha\left(x^{-\beta}-b^{-\beta}\right)}\right)\right]^{-2}} \mathrm{~d} x \\
& =\int_{0}^{b} x^{-r-\beta-1} \cdot \gamma \alpha \beta \mathrm{e}^{-\alpha\left(x^{-\beta}-b^{-\beta}\right)} \sum_{j=0}^{\infty}(j+1) \bar{\gamma}^{j}\left(1-\mathrm{e}^{-\alpha\left(x^{-\beta}-b^{-\beta}\right)}\right)^{j} \mathrm{~d} x \\
& =\sum_{j=0}^{\infty}(j+1) \bar{\gamma}^{j} \cdot \gamma \alpha \beta \int_{0}^{b} x^{-r-\beta-1} \mathrm{e}^{-\alpha\left(x^{-\beta}-b^{-\beta}\right)}\left(1-\mathrm{e}^{-\alpha\left(x^{-\beta}-b^{-\beta}\right)}\right)^{j} \mathrm{~d} x \\
& =\gamma \alpha \beta \sum_{i=0}^{\infty}(j+1) \bar{\gamma}^{j} \cdot \phi_{r, b}(x),
\end{aligned}
$$

where

$$
\begin{aligned}
\phi_{r^{-1}, b}(x) & =\int_{0}^{b} x^{-r-\beta-1} \mathrm{e}^{-\alpha\left(x^{-\beta}-b^{-\beta}\right)}\left(1-\mathrm{e}^{-\alpha\left(x^{-\beta}-b^{-\beta}\right)}\right)^{j} \mathrm{~d} x \\
\phi_{r^{-1}, b}(x) & =\int_{0}^{b} x^{-r-\beta-1} \mathrm{e}^{-\alpha\left(x^{-\beta}-b^{-\beta}\right)} \sum_{i=0}^{\infty}\left(\begin{array}{l}
j \\
i
\end{array}\right)(-1)^{i} \mathrm{e}^{-\alpha i\left(x^{-\beta}-b^{-\beta}\right)} \mathrm{d} x \\
= & \sum_{i=0}^{\infty}\left(\begin{array}{l}
j \\
i
\end{array}\right)(-1)^{i} \int_{0}^{b} x^{-r-\beta-1} \mathrm{e}^{-\alpha(i+1)\left(x^{-\beta}-b^{-\beta}\right)} \mathrm{d} x
\end{aligned}
$$




$$
\begin{aligned}
& =\sum_{i=0}^{\infty}\left(\begin{array}{l}
j \\
i
\end{array}\right)(-1)^{i} \int_{0}^{b} x^{-r-\beta-1} \mathrm{e}^{-\alpha(i+1) x^{-\beta}} \mathrm{e}^{\alpha(i+1) b^{-\beta}} \mathrm{d} x \\
& =\sum_{i=0}^{\infty}\left(\begin{array}{l}
j \\
i
\end{array}\right)(-1)^{i} \mathrm{e}^{\alpha(i+1) b^{-\beta}} \int_{0}^{b} x^{-r-\beta-1} \mathrm{e}^{-\alpha(i+1) x^{-\beta}} \mathrm{d} x,
\end{aligned}
$$

setting

$$
\begin{gathered}
-\alpha(i+1) x^{-\beta}=t \\
x^{-\beta}=\frac{1}{\alpha(i+1)} t \\
x=\left(\frac{1}{\alpha(i+1)}\right)^{\frac{-1}{\beta}} t^{\frac{-1}{\beta}} \\
\mathrm{d} x=\left(\frac{1}{\alpha(i+1)}\right)^{\frac{-1}{\beta}}\left(\frac{-1}{\beta}\right) t^{\frac{-1}{\beta}-1} \mathrm{~d} t \\
x=0 \rightarrow t=0 \\
x=t=\alpha(i+1) b^{-\beta},
\end{gathered}
$$

hence (14) becomes as follows

$$
\begin{aligned}
& \phi_{r^{-1}, b}(x)=\sum_{i=0}^{\infty}\left(\begin{array}{l}
j \\
i
\end{array}\right)(-1)^{i} \mathrm{e}^{\alpha(i+1) b^{-\beta}} \int_{0}^{b} x^{-r-\beta-1} \mathrm{e}^{-\alpha(i+1) x^{-\beta}} \mathrm{d} x \\
& =\sum_{i=0}^{\infty}\left(\begin{array}{l}
j \\
i
\end{array}\right)(-1)^{i} \mathrm{e}^{\alpha(i+1) b^{-\beta}} \int_{0}^{\alpha(i+1) b^{-\beta}}\left(\left(\frac{1}{\alpha(i+1)}\right)^{\frac{-1}{\beta}} t^{\frac{-1}{\beta}}\right)^{-r-\beta-1} \\
& \times \mathrm{e}^{-t}\left(\frac{1}{\alpha(i+1)}\right)^{\frac{-1}{\beta}}\left(\frac{-1}{\beta}\right) t^{\frac{-1}{\beta}-1} \mathrm{~d} t \\
& =\sum_{i=0}^{\infty}\left(\begin{array}{l}
j \\
i
\end{array}\right)(-1)^{i} \mathrm{e}^{\alpha(i+1) b^{-\beta}}\left(\left(\frac{1}{\alpha(i+1)}\right)^{\frac{-1}{\beta}}\right)^{-r-\beta-1}\left(\frac{1}{\alpha(i+1)}\right)^{\frac{-1}{\beta}} \\
& \left.\times\left(\frac{-1}{\beta}\right)^{\alpha(i+1) b^{-\beta}} \int_{0}^{\frac{-1}{\beta}}\right)^{r-\beta-1} t^{\frac{-1}{\beta}-1} \mathrm{e}^{-t} \mathrm{~d} t \\
& =\sum_{i=0}^{\infty}\left(\begin{array}{l}
j \\
i
\end{array}\right)(-1)^{i} \mathrm{e}^{\alpha(i+1) b^{-\beta}}\left(\left(\frac{1}{\alpha(i+1)}\right)^{\frac{-1}{\beta}}\right)^{-r-\beta-1}\left(\frac{1}{\alpha(i+1)}\right)^{\frac{-1}{\beta}} \\
& \times\left(\frac{-1}{\beta}\right)^{\alpha(i+1) b^{-\beta}} t_{0}^{\frac{-r+\beta}{\beta}-1} \mathrm{e}^{-t} \mathrm{~d} t \\
& =\sum_{i=0}^{\infty}\left(\begin{array}{l}
j \\
i
\end{array}\right)(-1)^{i} \mathrm{e}^{\alpha(i+1) b^{-\beta}}\left(\left(\frac{1}{\alpha(i+1)}\right)^{\frac{-1}{\beta}}\right)^{-r-\beta-1}\left(\frac{1}{\alpha(i+1)}\right)^{\frac{-1}{\beta}} \\
& \times\left(\frac{-1}{\beta}\right)\left(\alpha(i+1) b^{-\beta}\right)^{\frac{-r+\beta}{\beta}}
\end{aligned}
$$




$$
\times \Gamma\left(\frac{-r+\beta}{\beta}\right) \mathrm{e}^{\alpha(i+1) b^{-\beta}} \sum_{k=0}^{\infty} \frac{\left(\alpha(i+1) b^{-\beta}\right)^{k}}{\Gamma\left(\frac{-r+\beta}{\beta}+\alpha(i+1) b^{-\beta}+1\right)},
$$

where

$$
\begin{aligned}
& \int_{0}^{\alpha(i+1) b^{-\beta}} t^{\frac{-r+\beta}{\beta}-1} \mathrm{e}^{-t} \mathrm{~d} t \\
= & \left(\alpha(i+1) b^{-\beta}\right)^{\frac{-r+\beta}{\beta}} \Gamma\left(\frac{-r+\beta}{\beta}\right) \mathrm{e}^{\alpha(i+1) b^{-\beta}} \sum_{k=0}^{\infty} \frac{\left(\alpha(i+1) b^{-\beta}\right)^{k}}{\Gamma\left(\frac{-r+\beta}{\beta}+\alpha(i+1) b^{-\beta}+1\right)},
\end{aligned}
$$

by using the definition of the incomplete gamma function

$$
\gamma(s, x)=\int_{0}^{x} t^{s-1} \mathrm{e}^{-t} \mathrm{~d} t=x^{s} \Gamma(s) \mathrm{e}^{-x} \sum_{k=0}^{\infty} \frac{x^{k}}{\Gamma(s+x+1)},
$$

so the inverse $r$ th moments $\mu_{r^{-1}}^{\prime}$ about MORTFIWD

$$
\begin{aligned}
\mu_{r^{-1}}^{\prime}= & \gamma \alpha \beta \sum_{i=0}^{\infty}(j+1) \bar{\gamma}^{j} \sum_{i=0}^{\infty}\left(\begin{array}{l}
j \\
i
\end{array}\right)(-1)^{i} \mathrm{e}^{\alpha(i+1) b^{-\beta}}\left(\left(\frac{1}{\alpha(i+1)}\right)^{\frac{-1}{\beta}}\right)^{-r-\beta-1} \\
& \times\left(\frac{1}{\alpha(i+1)}\right)^{\frac{-1}{\beta}}\left(\frac{-1}{\beta}\right)\left(\alpha(i+1) b^{-\beta}\right)^{\frac{-r+\beta}{\beta}} \\
& \times \Gamma\left(\frac{-r+\beta}{\beta}\right) \mathrm{e}^{\alpha(i+1) b^{-\beta}} \sum_{k=0}^{\infty} \frac{\left(\alpha(i+1) b^{-\beta}\right)^{k}}{\Gamma\left(\frac{-r+\beta}{\beta}+\alpha(i+1) b^{-\beta}+1\right)} \\
= & \left.\gamma \alpha \beta \sum_{i=0}^{\infty} \sum_{i=0}^{\infty} \sum_{k=0}^{\infty}(-1)^{i+1}(j+1) \bar{\gamma}^{j}\left(\frac{1}{\alpha(i+1)}\right)^{\frac{-1}{\beta}}\right)^{-r-\beta-1} \\
& \times\left(\frac{1}{\alpha(i+1)}\right)^{\frac{-1}{\beta}}\left(\alpha(i+1) b^{-\beta}\right)^{\frac{-r+\beta}{\beta}} \\
& \times \Gamma\left(\frac{-r+\beta}{\beta}\right) \mathrm{e}^{2 \alpha(i+1) b^{-\beta}}\left(\begin{array}{l}
j \\
i
\end{array}\right) \frac{\left(\alpha(i+1) b^{-\beta}\right)^{k}}{\Gamma\left(\frac{-r+\beta}{\beta}+\alpha(i+1) b^{-\beta}+1\right)} .
\end{aligned}
$$

\section{The Mean Time to Failure}

We need to compute the mean time to failure of the Marshall and Olkin of the right truncated Fréchet-inverted Weibull distribution (MORTFIWD). If $X$ a random variable has the distribution MORTFIWD where $\theta=(\alpha, \beta, b)$ and $\gamma$ is a positive integer, then the mean time to failure of MORTFIWD is given by

$$
\operatorname{MTTF}=E(X)=\int_{0}^{b} x g(x) \mathrm{d} x=\int R(x) \mathrm{d} x,
$$

putting $r=1$ in Equation (16), we find 


$$
\begin{aligned}
& \operatorname{MTTF}=\gamma \alpha \beta \sum_{i=0}^{\infty} \sum_{i=0}^{\infty} \sum_{k=0}^{\infty}(-1)^{i+1}(j+1) \gamma^{-j}\left(\left(\frac{1}{\alpha(i+1)}\right)^{\frac{-1}{\beta}}\right)^{-\beta}\left(\frac{1}{\alpha(i+1)}\right)^{\frac{-1}{\beta}} \\
& \times\left(\alpha(i+1) b^{-\beta}\right)^{\frac{\beta-1}{\beta}} \Gamma\left(\frac{\beta-1}{\beta}\right) \mathrm{e}^{2 \alpha(i+1) b^{-\beta}}\left(\begin{array}{l}
j \\
i
\end{array}\right) \frac{\left(\alpha(i+1) b^{-\beta}\right)^{k}}{\Gamma\left(\frac{\beta-1}{\beta}+\alpha(i+1) b^{-\beta}+1\right)} \\
& =\gamma \alpha \beta \sum_{i=0}^{\infty} \sum_{i=0}^{\infty} \sum_{k=0}^{\infty}(-1)^{i+1}(j+1) \gamma^{-j}\left(\left(\frac{1}{\alpha(i+1)}\right)^{\frac{-1}{\beta}}\right)^{-\beta}\left(\frac{1}{\alpha(i+1)}\right)^{\frac{-1}{\beta}} \\
& \times\left(\alpha(i+1) b^{-\beta}\right)^{\frac{\beta-1}{\beta}} \frac{1}{\beta} \mathrm{e}^{2 \alpha(i+1) b^{-\beta}}\left(\begin{array}{l}
j \\
i
\end{array}\right) \frac{\left(\alpha(i+1) b^{-\beta}\right)^{k}}{\Gamma\left(\frac{\beta-1}{\beta}+\alpha(i+1) b^{-\beta}+1\right)} \\
& =\gamma \alpha \sum_{i=0}^{\infty} \sum_{i=0}^{\infty} \sum_{k=0}^{\infty}(-1)^{i+1}(j+1) \gamma^{-j}\left(\left(\frac{1}{\alpha(i+1)}\right)^{\frac{-1}{\beta}}\right)^{-\beta}\left(\frac{1}{\alpha(i+1)}\right)^{\frac{-1}{\beta}} \\
& \times\left(\alpha(i+1) b^{-\beta}\right)^{\frac{\beta-1}{\beta}} \mathrm{e}^{2 \alpha(i+1) b^{-\beta}}\left(\begin{array}{l}
j \\
i
\end{array}\right) \frac{\left(\alpha(i+1) b^{-\beta}\right)^{k}}{\Gamma\left(\frac{\beta-1}{\beta}+\alpha(i+1) b^{-\beta}+1\right)} \\
& =\gamma \alpha \sum_{i=0}^{\infty} \sum_{i=0}^{\infty} \sum_{k=0}^{\infty}(-1)^{i+1}(j+1) \gamma^{-j}\left(\frac{1}{\alpha(i+1)}\right)^{\frac{\beta-1}{\beta}} \alpha(i+1) b^{1-\beta} \\
& \times \mathrm{e}^{2 \alpha(i+1) b^{-\beta}}\left(\begin{array}{l}
j \\
i
\end{array}\right) \frac{\left(\alpha(i+1) b^{-\beta}\right)^{k}}{\Gamma\left(\frac{\beta-1}{\beta}+\alpha(i+1) b^{-\beta}+1\right)} \\
& =\gamma \alpha \sum_{i=0}^{\infty} \sum_{i=0}^{\infty} \sum_{k=0}^{\infty}(-1)^{i+1}(j+1) \gamma^{-j}\left(\begin{array}{l}
j \\
i
\end{array}\right)(\alpha(i+1))^{\frac{1}{\beta}} \\
& \times b^{1-\beta} \mathrm{e}^{2 \alpha(i+1) b^{-\beta}} \frac{\left(\alpha(i+1) b^{-\beta}\right)^{k}}{\Gamma\left(\frac{\beta-1}{\beta}+\alpha(i+1) b^{-\beta}+1\right)} \\
& =\gamma \alpha \sum_{i=0}^{\infty} \sum_{i=0}^{\infty} \sum_{k=0}^{\infty}(-1)^{i+1} \gamma^{-j}\left(\begin{array}{c}
j+1 \\
i
\end{array}\right)(\alpha(i+1))^{\frac{1}{\beta}} b^{1-\beta} \\
& \times \mathrm{e}^{2 \alpha(i+1) b^{-\beta}} \frac{\left(\alpha(i+1) b^{-\beta}\right)^{k}}{\Gamma\left(\frac{\beta-1}{\beta}+\alpha(i+1) b^{-\beta}+1\right)} .
\end{aligned}
$$

If $X$ a random variable has the distribution MORTFIWD. We find the mean time to failure of the Marshall and Olkin of the right truncated Fréchet-inverted Weibull distribution (MORTFIWD) 


$$
\begin{aligned}
\text { MTTF }= & \gamma \alpha \sum_{i=0}^{\infty} \sum_{i=0}^{\infty} \sum_{k=0}^{\infty}(-1)^{i+1} \bar{\gamma}^{j}\left(\begin{array}{c}
j+1 \\
i
\end{array}\right)(\alpha(i+1))^{\frac{1}{\beta}} b^{1-\beta} \mathrm{e}^{2 \alpha(i+1) b^{-\beta}} \\
& \times \frac{\left(\alpha(i+1) b^{-\beta}\right)^{k}}{\Gamma\left(\frac{\beta-1}{\beta}+\alpha(i+1) b^{-\beta}+1\right)} .
\end{aligned}
$$

\section{Maximum Likehood Method}

The maximum likelihood assessment method is essentially used and extends the maximum acquaintance about the properties of the assessment parameters. Furthermore, the natural approximation of the estimators may truthfully and mathematically for huge sample theory. Accordingly, the Maximum likehood assessment has depended to guess the unknown parameters $(\alpha, \beta$ and $b)$ of the MORTFIWD distribution. Let random variable $X$ from the observed distribution and have the parameters $(\alpha, \beta \text { and } b)^{\mathrm{T}}$ with size $n$. The sample likelihood function is

$$
\begin{aligned}
& L(\alpha, \beta, b)=\prod_{i=1}^{n} g\left(x_{i}\right) \\
& =\prod_{i=1}^{n} \frac{\gamma \alpha \beta \mathrm{e}^{-\alpha\left(x_{i}^{-\beta}-b^{-\beta}\right)}}{x_{i}^{\beta+1}\left[1-\bar{\gamma}\left(1-\mathrm{e}^{-\alpha\left(x_{i}^{-\beta}-b^{-\beta}\right)}\right)\right]^{2}} \\
& =\frac{\gamma^{n} \alpha^{n} \beta^{n} \mathrm{e}^{-\alpha \sum_{i=0}^{n}\left(x_{i}^{-\beta}-b^{-\beta}\right)}}{\prod_{i=1}^{n} x_{i}^{\beta+1} \prod_{i=1}^{n}\left[1-\bar{\gamma}\left(1-\mathrm{e}^{-\alpha\left(x_{i}^{-\beta}-b^{-\beta}\right)}\right)\right]^{2}} .
\end{aligned}
$$

The log-likehood function is

$$
\begin{aligned}
\log L(\alpha, \beta, b)= & n \log \gamma+n \log \alpha+n \log \beta-\alpha \sum_{i=1}^{n}\left(x_{i}^{-\beta}-b^{-\beta}\right) \\
& -\sum_{i=1}^{n} \log x_{i}^{\beta+1}-\sum_{i=1}^{n} \log \left[1-\bar{\gamma}\left(1-\mathrm{e}^{-\alpha\left(x_{i}^{-\beta}-b^{-\beta}\right)}\right)\right]^{2} \\
= & n \log \gamma+n \log \alpha+n \log \beta-\alpha \sum_{i=1}^{n}\left(x_{i}^{-\beta}-b^{-\beta}\right)-(\beta+1) \sum_{i=1}^{n} \log x_{i} \\
& -2 \sum_{i=1}^{n} \log \left[1-\bar{\gamma}\left(1-\mathrm{e}^{-\alpha\left(x_{i}^{-\beta}-b^{-\beta}\right)}\right)\right] .
\end{aligned}
$$

\section{Application}

The potentiality and flexibility of the new distribution is introduced in this section. The new model has contrasted with some other existing life-time models such as:

(i) Hinkley [10] introduced the first real-life data. Data compounds on 30 notices of the March precipitation in Minneapolis/St Paul. The values are 


$\begin{array}{llllllllllll}0.77 & 1.74 & 0.81 & 1.20 & 1.95 & 1.20 & 0.47 & 1.43 & 3.37 & 2.20 & 2.81 & 1.87 \\ 3.00 & 3.09 & 1.51 & 2.10 & 0.52 & 1.62 & 1.31 & 0.32 & 0.59 & 0.81 & 1.18 & 1.35 \\ 4.75 & 2.48 & 0.96 & 1.89 & 0.90 & 2.05 & & & & & & \end{array}$

(ii) The second data also compounds on 30 values for the failure time which be repairable objects used by Murthy et al. [11]. The values are

$\begin{array}{llllllllll}1.43 & 0.11 & 0.71 & 0.77 & 2.63 & 1.49 & 3.46 & 2.46 & 0.59 & 1.97 \\ 0.74 & 1.23 & 0.94 & 4.36 & 0.40 & 1.74 & 4.73 & 2.23 & 0.45 & 1.86 \\ 0.70 & 1.06 & 1.46 & 0.30 & 1.82 & 2.37 & 0.63 & 1.23 & 1.24 & 1.17\end{array}$

(iii) Bhaumik [12] introduced the third real data life The values are

$\begin{array}{llllllllllll}5.1 & 1.2 & 1.3 & 0.6 & 0.5 & 2.4 & 0.5 & 1.1 & 8.0 & 0.8 & 0.4 & 0.6 \\ 0.9 & 0.4 & 2.0 & 0.5 & 5.3 & 3.2 & 2.7 & 2.9 & 2.5 & 2.3 & 1.0 & 0.2 \\ 0.10 & 0.1 & 1.8 & 0.9 & 2.0 & 4.0 & 6.8 & 1.2 & 0.4 & 0.2 & & \end{array}$

The new generated Marshall and Olkin of the right truncated Fréchet-inverted Weibull distribution (MORTFIWD) is compared with the right truncated Fréchet-inverted Weibull distribution, Marshall-Olkin extended inverted $\mathrm{Ku}$ maraswamy (MOEIK) distribution with the Generalized Inverted Kumaraswamy (GIK), Transmuted Exponentiated Inverse Rayleigh (TEIR), Logistic Weibull (LW), Transmuted Power Lindley (TPL), Marshall Olkin Frechet (MOFr) and Inverted Kumaraswamy (IK) distribution for these data sets.

The new distribution is compared with other distributions. By using some numerous fineness of fit measures such as the log likelihood function (2l), Akaike Information Criterion (AIC), Bayesian Information Criterion (BIC), Consistent Akaike Information Criterion(CAIC), Hanna-Quinn Information Criterion (HQIC). The assessment parameters and fineness of fit measures of the pervious real life data sets. The fineness of fit measures such as AIC, BIC, CAIC, HQIC [13].

The guess of maximum likehood method for parameters with the fineness of fit measures (March precipitation).

\begin{tabular}{lllllllll} 
Models & \multicolumn{2}{l}{ Estimates } & & -21 & AIC & BIC & CAIC & HQIC \\
MORTFIWD $(\alpha, \beta, b)$ & 13.320 & 1.185136 & 4.75 & 75.0588 & 81.0589 & 81.982 & 85.2625 & 82.3036 \\
RTFIWD $(\alpha, \beta)$ & 1.38587 & 1.14316 & - & 75.7754 & 81.7754 & 82.6985 & 85.975 & 83.1202 \\
MOEIK $(\alpha, \beta, \lambda)$ & 4.3228 & 6.5798 & 6.9226 & 76.69 & 82.69 & 86.89 & 84.79 & 84.03 \\
GIK $(\alpha, \beta, \gamma)$ & 1.9552 & 3.9501 & 1.4202 & 78.63 & 84.63 & 88.84 & 86.77 & 85.98 \\
TEIR $(\alpha, \theta, \lambda)$ & 6.5630 & 0.0958 & -0.6700 & 84.20 & 90.20 & 94.41 & 92.30 & 91.55 \\
LW $(\alpha, \beta, \lambda)$ & 2.7709 & 0.3635 & 1.0061 & 77.86 & 83.86 & 88.06 & 85.96 & 85.21 \\
TPL $(\alpha, \theta, \lambda)$ & 1.5965 & 0.4801 & 0.5812 & 77.30 & 83.31 & 89.05 & 86.18 & 86.19 \\
MOFr $(\alpha, \beta, \sigma)$ & 42.598 & 2.6975 & 0.3548 & 77.60 & 83.60 & 87.80 & 85.70 & 84.94 \\
IK $(\alpha, \beta)$ & 2.9872 & 8.5899 & - & 78.85 & 83.85 & 87.65 & 85.25 & 84.74
\end{tabular}


The guess of maximum likehood method for parameters with the fineness of fit measures (failure time data).

\begin{tabular}{lllllllll} 
Models & \multicolumn{2}{l}{ Estimates } & & -21 & AIC & BIC & CAIC & HQIC \\
MORTFIWD $(\alpha, \beta, b)$ & 30.3832 & 0.0614346 & 4.73 & 76.9488 & 82.9488 & 87.5279 & 83.7488 & 84.5104 \\
RTFIWD $(\alpha, \beta)$ & 1.39561 & 0.680631 & - & 79.1164 & 85.1164 & 89.32 & 86.0395 & 86.4611 \\
MOEIK $(\alpha, \beta, \lambda)$ & 3.8031 & 2.1861 & 9.6185 & 79.60 & 85.60 & 89.80 & 87.70 & 86.94 \\
GIK $(\alpha, \beta, \gamma)$ & 1.1623 & 1.4462 & 1.8481 & 81.64 & 87.64 & 91.84 & 89.74 & 88.98 \\
$\operatorname{TEIR}(\alpha, \theta, \lambda)$ & 7.2940 & 0.0254 & -0.8803 & 117.0 & 123.0 & 127.2 & 125.1 & 124.3 \\
$\operatorname{LW}(\alpha, \beta, \lambda)$ & 2.8927 & 0.5531 & 0.07775 & 80.83 & 86.83 & 91.03 & 88.93 & 88.17 \\
$\operatorname{TPL}(\alpha, \theta, \lambda)$ & 1.3380 & 0.6301 & 0.5356 & 80.73 & 86.73 & 90.93 & 88.83 & 88.07 \\
$\operatorname{MOFr}(\alpha, \beta, \sigma)$ & 63.165 & 2.1070 & 0.1669 & 81.53 & 87.53 & 91.73 & 89.63 & 88.87 \\
$\operatorname{IK}(\alpha, \beta)$ & 2.4609 & 4.1716 & - & 82.48 & 86.48 & 90.28 & 88.88 & 87.37
\end{tabular}

The guess of maximum likehood method for parameters with the fineness of fit measures (chloride data).

$\begin{array}{lllllllll}\text { Models } & \text { Estimates } & & & -21 & \text { AIC } & \text { BIC } & \text { CAIC } & \text { HQIC } \\ \text { MORTFIWD }(\alpha, \beta, b) & 2.27479 & 0.401845 & 8 & 106.265 & 112.265 & 113.065 & 116.844 & 113.826 \\ \text { RTFIWD }(\alpha, \beta) & 1.09489 & 0.602397 & - & 106.279 & 112.28 & 113.08 & 116.859 & 113.841 \\ \text { MOEIK }(\alpha, \beta, \lambda) & 2.1193 & 1.7026 & 2.2471 & 110.9 & 116.9 & 121.5 & 119.2 & 118.5 \\ \text { GIK }(\alpha, \beta, \gamma) & 2.0236 & 2.6369 & 0.8858 & 111.5 & 117.5 & 122.1 & 119.8 & 119.1 \\ \text { TEIR }(\alpha, \theta, \lambda) & 6.9784 & 0.0138 & -0.7798 & 170.8 & 176.8 & 181.4 & 179.1 & 178.3 \\ \operatorname{LW}(\alpha, \beta, \lambda) & 2.7709 & 0.7056 & 0.5526 & 111.9 & 117.9 & 122.5 & 120.2 & 119.5 \\ \operatorname{TPL}(\alpha, \theta, \lambda) & 0.9265 & 0.7453 & 0.4094 & 111.2 & 117.2 & 122.5 & 120.5 & 119.7 \\ \operatorname{MOFr}(\alpha, \beta, \sigma) & 29.053 & 1.4730 & 0.1124 & 111.4 & 117.4 & 121.9 & 119.7 & 119.3 \\ \operatorname{IK}(\alpha, \beta) & 1.7409 & 2.1058 & - & 112.5 & 117.5 & 122.6 & 120.1 & 119.6\end{array}$

\section{Conclusion}

A new probability distribution is introduced by using Marshall and Olkin transformation. Some of its properties such as moments, moment generating function, order statistics and reliability functions are derived. The method of maximum likelihood is used to estimate the model parameters.

\section{Conflicts of Interest}

The authors declare no conflicts of interest regarding the publication of this paper.

\section{References}

[1] Dubey, S.D. (1970) Compound Gamma, Beta and F Distribution. Metrika, 16, 27-31. https://doi.org/10.1007/BF02613934

[2] Voda, V.G. (1972) On the Inverse Rayleigh Distributed Random Varaible. Reports of Statistical Application Research, 19, 13-21.

[3] Calabria, R. and Pulcini, G. (1990) On the Maximum Likehood and Least-Squares 
Estimation in the Inverse Weibull Distributions. Statistics Applicata, 2, 53-66.

[4] Dey, S. (2007) Inverted Exponential Distribution as a Life Distribution as Model from a Bayesian Viewpoint. Data Science Journal, 6, 107-113.

https://doi.org/10.2481/dsj.6.107

[5] Rosaiah, K. and Kantam, R.R.L. (2005) Acceptance Sampling Based on the Inverse Rayleigh Distribution. Stochastics and Quality Control, 20, 277-286. https://doi.org/10.1515/EQC.2005.277

[6] Sharma, V.K., Singh, U. and Agiwal, V. (2015) The Inverse Lindly Distribution: A Stress-Strength Relibility model with Application to Head and Neck Cancer Data. Journal of Industrial and Production Engineering, 32, 162-173. https://doi.org/10.1080/21681015.2015.1025901

[7] Fréchet, M. (1927) Sur la loi de Probabilité de l'écart Maximum. Annales de la Société Polonaise de Mathematique, 6, 93-116.

[8] Haq, M.A., Usman, R.M., Hashmi, S. and Al-Omeri, A.I. (2019) The Marshall-Olkin Length-Biased Exponential Distribution and Its Applications. Journal of King Saud University-Science, 31, 246-251. https://doi.org/10.1016/j.jksus.2017.09.006

[9] Marshall, A.W. and Olkin, I. (1997) A New method for Adding a Parameter to a Family of Distributions with Application to the Exponential and Weibull Families. Biometrika, 84, 641-652. https://doi.org/10.1093/biomet/84.3.641

[10] Hinkley, D. (1977) On Quick Choice of Power Transformation. Journal of the Royal Statistical Society, Series C, 26, 67-69. https://doi.org/10.2307/2346869

[11] Murthy, D.N.P., Xie, M. and Jiang, R. (2004) Weibull Models. John Wily \& Sons, New York.

[12] Bhaumik, D.K., Kapur, K. and Gibbons, R.D. (2009) Testing Parameters of Agamma Distribution for Small Samples. Technometrics, 51, 326-334.

https://doi.org/10.1198/tech.2009.07038

[13] Usman, R.M. and Ahsan ul Haq, M. (2020) The Marshall-Olkin Extended Inverted Kumaraswamy Distribution Theory and Applications. Journal of King Saud University-Science, 32, 356-365. https://doi.org/10.1016/j.jksus.2018.05.021 